malignant germ cell tumors have no complete intra peritoneal surgical staging.

\section{EPV195/\#37 VEGF-A INHIBITOR INDUCED TUMOR-ASSOCIATED MACROPHAGE REPROGRAMMING AND PD-L1 OVEREXPRESSION VIA A DUAL ROLE OF IFN- $\Gamma$ IN OVARIAN CANCER}

${ }^{1,2} \mathrm{C} \mathrm{Li*}{ }^{*},{ }^{1,2}$ J Peng, ${ }^{3} \mathrm{Y} \mathrm{Li},{ }^{1,2} \mathrm{C}$ Sun, ${ }^{4} \mathrm{Z}$ Zhang, ${ }^{1,2} \mathrm{~L} \mathrm{Liu},{ }^{1,3} \mathrm{~S} Y a 0,{ }^{1,2} \mathrm{Li}_{1}{ }^{1,2} \mathrm{~K}$ Song. ${ }^{1}$ Qilu Hospital of Shandong University, Gynecology Oncology Key Laboratory, Jinan, China; ${ }^{2}$ Qilu Hospital, Cheeloo College of Medicine, Shandong University, Obstetrics and Gynecology, Jinan, China; ${ }^{3}$ School of Medicine, Cheeloo College of Medicine, Shandong University, Obstetrics and Gynecology, Jinan, China; ${ }^{4}$ Beijing Chao-Yang Hospital, Capital Medical University, Obstetrics and Gynecology, Beijing, China

\subsection{6/ijgc-2021-IGCS.266}

Objectives The beneficial effects of vascular endothelial growth factor A (VEGF-A) inhibitor are only observed in a subset of patients with advanced ovarian cancer. The lack of response of VEGF-A inhibitors is thought to be related with the immunosuppressive microenvironment; however, this is still controversial. A better understanding of the underlying mechanism of VEGF-A inhibitor (Bevacizumab)-mediated the immune escape could benefit the development of therapeutic regimens for patients with ovarian cancer.

Methods The polarization of tumor-associated macrophages (TAMs), IFN- $\gamma$ secretion of macrophages and ovarian cancer cells, PD-L1 expression in ovarian cancer cells, and phagocytic function of macrophages after Bevacizumab intervention were examined. In addition, the efficacy of combined Bevacizumab with anti-PD-1 antibody (aPD-1) was evaluated in a murine ovarian cancer model.

Results We first identified that Bevacizumab stimulated IFN- $\gamma$ secretion from macrophages and ovarian cancer cells. Moreover, we demonstrated that Bevacizumab upregulated PD-L1 expression in an IFN- $\gamma$-PI3K-NF- $\mathrm{BB}-$ dependent manner in ovarian cancer. Interestingly, although Bevacizumab elicited antitumor immunity by inducing macrophage polarization to an M1 phenotype via elevated IFN- $\gamma$ secretion, the phagocytic function of macrophages was suppressed by upregulated PD-1 expression in macrophages. Furthermore, Bevacizumab combined with an aPD-1 significantly decreased the tumor burden and prolonged survival time in mice with ovarian cancer.

Conclusions Here, we demonstrated a dual role of IFN- $\gamma$ induced by Bevacizumab in ovarian cancer. Specifically, IFN- $\gamma$ promoted immune activation in terms of M1 polarization and immune suppression through PD-L1/PD-1 upregulation. The combination of Bevacizumab and aPD-1 improved the local immune status and provided a promising novel therapeutic regimen against ovarian cancer.

\section{EPV196/\#377 PROGESTERONE TRIGGERS CARCINOGENESIS IN A MOUSE MODEL THAT PHENOCOPIES HIGH GRADE SEROUS CARCINOMA HARBORING A GERMLINE BRCA MUTATION}

${ }^{1} \mathrm{C}-\mathrm{H}$ Cho*, ${ }^{2} \mathrm{SY}$ Kwon, ${ }^{1} \mathrm{~S}$-J Shin, ${ }^{1} \mathrm{~S}$ Lee, ${ }^{3} \mathrm{~J}$ Kim. ${ }^{1}$ Keimyung University Dongsan Hospital, Department of Obstetrics and Gynecology, Daegu, Korea, Republic of; ${ }^{2}$ Keimyung University Dongsan Hospital, Department of Pathology, Daegu, Korea, Republic of; Indiana University Melvin and Bren Simon Comprehensive Cancer Center, Department of Biochemistry and Molecular Biology, Indianapolis, USA

10.1136/ijgc-2021-IGCS.267
Objectives The purpose of this study is elucidating the role of ovarian hormones in the development of ovarian cancer.

Methods To evaluate the effects of steroid hormones on HGSC development, upon ovariectomy at 5-6 weeks of age, DKO mice were implanted subcutaneously with a pellet of $17-$ $\beta$ estradiol (E2) (0.72 mg/90days/mouse), progesterone (P4) (25 mg/90 days/mouse), or combined E2 (0.72 mg) \& P4 (25 $\mathrm{mg})$. Also, for shorter periods of P4 exposure, ovariectomized DKO mice were treated with a P4 pellet of $2 \mathrm{mg}$ (1 week) or $6 \mathrm{mg}$ (3 weeks). Another set of ovariectomized DKO mice implanted with a placebo served as controls. To examine whether mifepristone (RU486) inhibits HGSC development in DKO mice by blocking PR, DKO mice with intact ovaries were implanted with a mifepristone pellet once at $9 \mathrm{mg} / 90$ days (3 months) or three times at $33.3 \mathrm{mg} / 60$ days. For a control group, DKO mice received a placebo pellet for mifepristone.

Results We show that ovarian progesterone is a crucial endogenous factor inducing the development of primary tumors progressing to metastatic ovarian cancer in a mouse model of high- grade serous carcinoma (HGSC), the most common and deadliest ovarian cancer type. Blocking progesterone signaling by the pharmacologic inhibitor mifepristone or by genetic deletion of the progesterone receptor (PR) effectively suppressed HGSC development and its peritoneal metastases. Strikingly, mifepristone treatment profoundly improved mouse survival ( $\sim 18$ human years).

Conclusions Targeting progesterone/PR signaling could offer an effective chemopreventive strategy, particularly in high-risk populations of women carrying a deleterious mutation in the BRCA gene.

\section{EPV197/\#394 BCARE- FUNCTIONALLY ASSESSING TREATMENT RESPONSE IN OVARIAN CANCER PATIENTS}

\footnotetext{
${ }^{1,2} \mathrm{~F}$ Geissler* ${ }^{2} \mathrm{R}$ Coelho, ${ }^{1,2} \mathrm{~T}$ Zwimpfer, ${ }^{2} \mathrm{~A}$ Fedier, ${ }^{2} \mathrm{~F}$ Jacob, ${ }^{1,2}{ }^{2} \mathrm{~V}$ Heinzelmann-Schwarz. 'University Hospital Basel, Department of Gynaecology and Obstetrics, Basel, Switzerland; ${ }^{2}$ University of Basel, Department of Biomedicine, Basel, Switzerland
}

\subsection{6/ijgc-2021-IGCS.268}

Objectives Treatment regimens in oncology frequently become personalized and multimodal. Therefore, reliable biomarkers to predict drug responses are needed. Here, we establish a functional assay reflecting response to DNA damage-based treatment approaches.

Methods Our Bimodal prediction of ovarian CAncer treatment REsponse (BCARE) score combines irradiation-induced DNA damage with functional assessment of homologous recombination (HR) DNA repair visualized by immunofluorescence detection of $\gamma \mathrm{H} 2 \mathrm{AX}$ and cyclinA2/RAD51 positive cells. BCARE is quantified by automated image analysis using Image $\mathrm{J}$ and $\mathrm{R} /$ Bioconductor. Treatment response of cancer cells to PARP inhibition, radiotherapy, and chemotherapy is analyzed by colony formation and MTT cell viability assays.

Results BCARE reflects the percentage of RAD51/cyclinA2 double positive cells. It significantly correlated with response to various drugs tested in 6 ovarian cancer cell lines: olaparib $(\mathrm{R}=0.92, \quad \mathrm{p}=0.0095), \quad$ radiotherapy $\quad(\mathrm{R}=0.78$, $\mathrm{p}=0.0374), \quad$ cisplatin $(\mathrm{R}=0.85, \quad \mathrm{p}=0.0325), \quad$ doxorubicin $(\mathrm{R}=0.92, \mathrm{p}=0.0095)$, and carboplatin $(\mathrm{R}=0.77, \mathrm{p}=0.0738)$. Additionally, we show that the BCARE coincides with the 
cisplatin-resistant phenotype of A2780 cell line (BCARE A2780 3.3\% versus 39\% in A2780-cisplatin-resistant cells). The assay currently tests ex vivo patient derived cultures using a retrospective cohort of ovarian cancer patients. Correlations between BCARE scores and patient response to treatment will be investigated.

Conclusions Our BCARE-Score is capable of identifying dynamic alterations in HR-pathways as indicated by the differences observed in A2780 and cisplatin-resistant subline, which is not assessed by the current clinically applied HR assays. BCARE shows clear potential to be an effective tool in the prediction of primary drug response and more importantly in the detection of developed drug resistance.

\section{EPV198/\#424 LAPAROSCOPIC RESTAGING SURGERY FOR GYNAECOLOGICAL MALIGNANCIES}

${ }^{1} \mathrm{O}$ Puga*, 'M Urzua, ${ }^{2}$ E Pertossi. ${ }^{1}$ Hospital Dr Sotero del Rio, Obstetric and Gynecology, Santiago, Chile; ${ }^{2}$ Pontificia Universidad Catolica de Chile, Obstetric and Gynecology, Santiago, Chile

\subsection{6/ijgc-2021-IGCS.269}

Objectives To evaluate the feasibility and safety of laparoscopically staging patients with previous incomplete staged gynaecological cancers

Methods Patients without presurgical evidence of metastatic disease were laparoscopically reassessed. The procedure involved para-aortic and pelvic lymph node dissection and omentectomy for ovarian, fallopian tube and endometrial carcinoma; exclusive pelvic lymph node dissection for cervical carcinoma, oophorectomy and omentectomy for borderline tumors. Medical records were reviewed.

Results We performed 51 laparoscopic restaging surgeries: 14 ovarian cancer, 15 endometrial cancer, 17 borderline ovarian tumors, 4 cervical cancers and one fallopian tube carcinoma. Mean age was 48 years (16-70). In 39 patients the first surgery was performed by laparotomy. The mean body mass index was $28(20-40)$. Operative room time was $203 \mathrm{~min}(70-390)$ and mean postoperative hospital stay was 2 days. We performed 32 pelvic lymphadenectomies (average 15 lymph nodes), 30 para-aortic lymphadenectomies (8 lymph nodes), 27 omentectomies and 17 hysterectomies. Average estimated blood lost was $85 \mathrm{cc}$. There was one laparo-conversion for adhesions, one bowel injury, one cardiorespiratory arrest at recovery room and 2 lymphatics cystics. Lymph nodes and omentum were negative for metastasis. There were no patients up staged, in 9 endometrial and 9 ovarian cancers the complete negative restaging allowed us to decide that adjuvant therapy was not necessary. Five patients received adjuvant radiotherapy and 5 chemotherapy

Conclusions Laparoscopy is a feasibility technical option to perform restaging of gynaecological malignancies. Decreasing hospital stay, postoperative pain, few blood lost and low morbidity. Laparotomy for adhesions and risk of visceral injury may be anticipated.

\section{EPV199/\#430 CLINICAL AND SURVIVAL OUTCOMES OF MALIGNANT NON-DYSGERMINOMATOUS GERM CELL TUMOR OF OVARY: A SINGLE INSTITUTIONAL EXPERIENCE OF 64 PATIENTS}

${ }^{1} \mathrm{H}$ Mansouri, ${ }^{2} \mathrm{H}$ Salhi, ${ }^{3,4}$ I Zemni ${ }^{*},{ }^{1} \mathrm{~L}$ Achouri, ${ }^{3,4} \mathrm{MA}$ Ayadi, ${ }^{3} \mathrm{~K}$ Rahal. ${ }^{1}$ Regional Hospital of Jendouba, University of Tunis ELManar Tunisia, Department of Surgical Oncology, Jendouba, Tunisia; ${ }^{2}$ Regional Hospital of Siliana, University of Tunis ELManar Tunisia, Department of Surgical Oncology, Siliana, Tunisia; ${ }^{3}$ Salah Azaiez Institute of Cancerology, University of Tunis El Manar, Department of Surgical Oncology, Tunis, Tunisia; ${ }^{4}$ University of Tunis El Manar, Sciences Faculty of Tunis, Laboratory of Microorganismes and Active Biomolecules, Tunis, Tunisia

\subsection{6/ijgC-2021-IGCS.270}

Objectives To evaluate clinicopathological features and survival outcomes of malignant non-dysgerminomatous germ cell tumor (MNDGCT) of the ovary.

Methods We retrospectively recorded clinicopathological and therapeutic data of 64 patients with MNDGCT of ovary treated at the Salah Azaiez Institute of Tunisia between 1970 and 2012.

Results The median age was 26 years (range7-75 years). The most frequent subtype was immature teratoma $(n=27$, $42.18 \%)$ followed by yolk-sac tumor $(n=15,23.43 \%)$ and mixed germ cell tumor $(n=11,17.18 \%)$. Most of the patients had stage I and II disease (41 cases, 64.1\%) while 17 (26.6\%) and $6(9.3 \%)$ were staged III and IV disease, respectively. Radical surgery was performed in 23 patients $(35.8 \%)$ and conservative surgery in 41 patients(64.2\%) associated with lymph node dissection in 19 cases. Complete macroscopic resection was obtained in 48 patients (78.68\%) and lymph node metastasis was observed in $41.5 \%$ of cases. Adjuvant chemotherapy was indicated in cases in $54.68 \%$ of cases. After a mean time follow-up of 74 months (7-182 months), complete remission was observed in 47 patients. The 5 -year progression-free survival (PFS) was $73.5 \%$.The 5 year overall survival (OS) was $82.23 \%$ and was significantly decreased in young patients $\leq 15$ years $(49.5 \%$ vs $89.4 \% ; \mathrm{p}=0.003)$, advanced stage $(94.6 \%$ in stage I-II vs $59.8 \%$ in stage III-IV; $\mathrm{p}=0.01)$ and macroscopic residual disease $(88.9 \%$ vs $52.9 \%$; $\mathrm{p}=0.02)$.No difference in OS was noted following stratification by tumor size $\leq$ or $>20 \mathrm{~cm}(84.7 \%$ vs $74.6 ; \mathrm{p}=0.44)$ and conservative or radical surgery $(89.8 \%$ vs $70 \% ; \mathrm{p}=0.34)$

Conclusions Macroscopic residual disease as well as advanced FIGO stage and age are the main prognostic factors in MNDGCT.

\section{EPV200/\#432 CLINICAL AND SURVIVAL OUTCOMES OF PURE DYSGERMINOMA OF OVARY: A SINGLE INSTITUTIONAL EXPERIENCE OF 31 PATIENTS}

${ }^{1} \mathrm{H}$ Salhi, ${ }^{2} \mathrm{H}$ Mansouri, ${ }^{3,4} \mathrm{MA}$ Ayadi, ${ }^{2} \mathrm{~L}$ Achouri, ${ }^{3,4} \mathrm{I}$ Zemni*, ${ }^{3} \mathrm{~K}$ Rahal. ${ }^{1}$ Regional Hospital of Siliana, University of tunis ELManar Tunisia, Department of Surgical Oncology, Siliana, Tunisia; ${ }^{2}$ Regional Hospital of Jendouba, University of tunis ELManar Tunisia, Department of Surgical Oncology, Jendouba, Tunisia; ${ }^{3}$ Salah Azaiez Institute of Cancerology, University of Tunis El Manar, Department of Surgical Oncology, Tunis, Tunisia; ${ }^{4}$ Sciences Faculty of Tunis, University of Tunis El Manar, Laboratory of Microorganismes and Active Biomolecules, Tunis, Tunisia

10.1136/ijgc-2021-IGCS.271 